\title{
Optimization method of navigation information mode under the condition of multi-exit city expressway
}

\author{
HongJian Zhao ${ }^{1, *}$ \\ ${ }^{1}$ school of traffic and transportation, 100044 Beijing Jiaotong University, Beijing
}

\begin{abstract}
In recent years, mobile phone navigation software has been widely used, but the voice broadcast information of many navigation software is not clear, which is easy to cause confused driving. Urban expressway is an important artery of the city. The road and traffic conditions of urban expressways are extremely complicated, and there are many types of exits, which can easily cause confused driving. Therefore, optimization of navigation modes under the condition of multi-exit urban expressways is particularly important. Based on the experimental data of simulated driving, this paper focuses on the influence of voice navigation broadcast timing on driving under urban expressway, founding driver's behavior is significantly different under diverse navigation modes. This paper uses the principal component analysis method to comprehensively evaluate the navigation broadcast timing, and gives the optimal navigation broadcast timings for various multi-exit urban expressways. In most cases, the first broadcast time should be $1500 \mathrm{~m}$ from the exit. This paper clarifies the impact of navigation broadcast timing on driving, and optimizes the navigation mode of multi-exit urban expressways, which is of great significance for reducing yaw under navigation and improving navigation services.
\end{abstract}

\section{Introduction}

The urban expressway is an important artery for the whole of city. The distribution of lanes and exits on urban expressways is complex, and there are many exits. Drivers driving on unfamiliar express roads need to rely on the reasonable guidance of Mobile navigation. Therefore, accurate and efficient navigation guidance is of great significance for driving on expressway.

The number of potential users of mobile navigation in my country is huge in the future. However, there are many problems between the mobile navigation and the driver. Problems such as untimely navigation broadcast timing will cause the driver distracted and confused. At present, there are many types of mobile navigation software in our country, with different navigation modes. Therefore, mobile navigation software urgently needs to be unified and standardized.

The navigation mode studied in this paper specifically refers to the timing of navigation broadcast. First, perform data cleaning, eliminate abnormal values, so as to extract 9 indexes that characterize driving behavior under different navigation broadcast timings. Second, based on driving behavior indexes, this paper studies the influence of different navigation broadcast timings on the behavior of drivers on multi-exit expressway. Third, the principle component analysis method is used to evaluate different navigation broadcast timings, so as to obtain the optimal navigation mode.

\section{Literature review}

The current research on navigation mainly includes the humanized design of navigation equipment and the influence of navigation on the driving.

The main functions of mobile navigation include graphic instruction interface and voice broadcast, which together provide drivers with reasonable guidance, but will interfere with the driver's driving attention, so the humanized research on navigation equipment Is very important. MinLu studied the importance of navigation users' cognitive psychology, colourful psychology and other psychological factors in the design of navigation software interface [1]. Zhu Wei studied the human factors design and evaluation method of navigation software interface to improve the usability level of car navigation software interface [2]. Wang Wei et al. proposed a series of safety design standards for vehicle information systems from the perspective of humancomputer interaction and driver's behavior [3].

Zhu Dan, Wu Ling, etc. studied the impact of vehicle-mounted information on the driver's blink characteristics and driving load, and found that navigation information increases the driver's driving load, but reduces the load when providing voice broadcast [4]. Meiping Yun uses driving simulation technology to study the impact of roadside navigation and in-vehicle navigation on driver's lane-changing behavior on expressway under three traffic flow

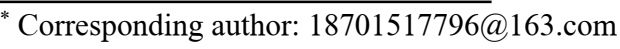


densities of medium, low, and high. The experiment found that the earlier the car navigation information is provided for the first time, the more fully prepared the driver is for changing lanes, and the better it is for driving comfort [5].

\section{Data description and methodology}

\subsection{Data base}

As a participant, I conducted a simulated driving experiment with the entire team. This experiment is mainly based on a driving simulator. Experimental scenes include 3-exit expressway, 2-exit expressway, and 4-exit expressway

The independent variable is different navigation broadcast timing, the dependent variable is the driving behavior index, namely the safety index: LP value, steering wheel angle value, braking times; ride comfort index: acceleration value, lane changing times, throttle value, braking value; efficiency Performance indicators: speed value, driving time. In each scenario, starting from $2000 \mathrm{~m}$ upstream of the expressway exit, all behavior indexes data was collected every $5 \mathrm{~m}$, for a total of 400 sets of behavior index data. timing:

There are three modes of navigation broadcast

Mode1: Broadcast at a distance of

$2000 \mathrm{~m} / 1500 \mathrm{~m} / 1000 \mathrm{~m} / 500 \mathrm{~m}$ from the exit;

Mode 2: Broadcast $1500 \mathrm{~m} / 1000 \mathrm{~m} / 500 \mathrm{~m}$ from the exit;

Mode 3: Broadcast at a distance of $1000 \mathrm{~m} / 500 \mathrm{~m}$ from the exit;

\subsection{The impact of navigation mode on multi-exit expressway on driving behavior}

First, the general linear model repeated variance measurement [6] method is used to explore whether different navigation timings have an impact on driving behavior indexes, that is, a significance analysis is performed.

Among the three types of indexes, since indexes of speed, LP value, and acceleration are the most representative, they are selected to represent various indexes for significant impact analysis. First, the average value of each index data in each scene is processed, Then test whether different navigation broadcast timings have significant effects on the these three indexes.

On the 2-exit,3-exit,4-exit expressway, Probability distributions of speed at the three navigation broadcast timings is shown in Figure 1

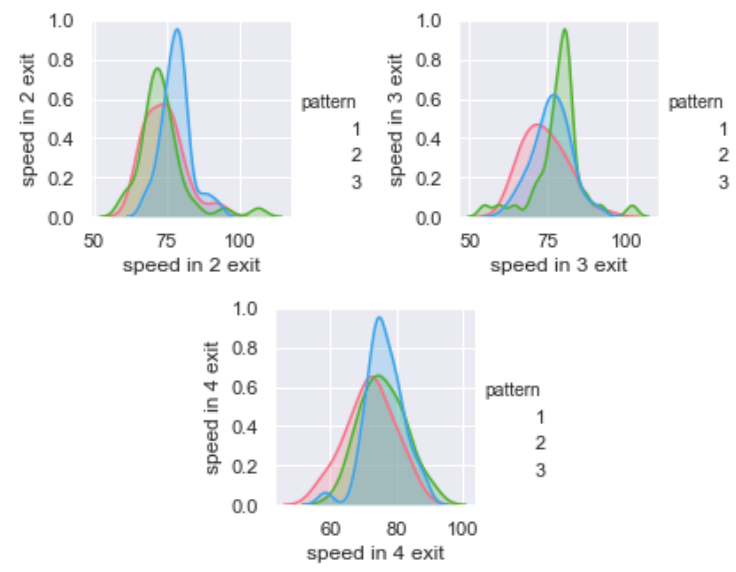

Fig 1 Probability distributions of speed

It can be seen from Table 1 that the navigation broadcast timing has a significant impact on the speed value, that is, the speed value has a significant difference under different navigation broadcast timings.

Table 1 The influence of broadcast timing on Speed

\begin{tabular}{|c|c|c|}
\hline \multirow{2}{*}{ speed } & $\begin{array}{l}\text { Mauchly's test of } \\
\text { sphericity }\end{array}$ & $\begin{array}{l}\text { Effect test (Navigation } \\
\text { broadcast timing) }\end{array}$ \\
\hline \multirow{2}{*}{ 2-exit } & 0.288 & 0.03 \\
\cline { 2 - 3 } & $\checkmark$ & Significant difference \\
\hline \multirow{2}{*}{ 3-exit } & 0.442 & 0.001 \\
\cline { 2 - 3 } & $\checkmark$ & Significant difference \\
\hline \multirow{2}{*}{ 4-exit } & 0.516 & 0.000 \\
\cline { 2 - 3 } & $\checkmark$ & Significant difference \\
\hline
\end{tabular}

On the 2-exit, 3-exit, 4-exit expressway, the probability distributions of acceleration at the three navigation broadcast timings is shown in Figure 2.

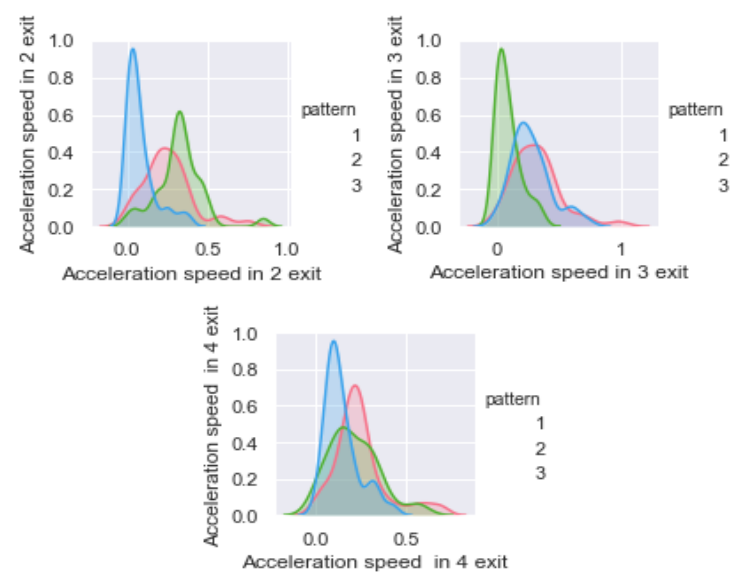

Fig 2 Probability distributions of acceleration

It can be seen from Table 2 that the navigation broadcast timing has a significant impact on acceleration, that is, the acceleration has a significant difference under different navigation broadcast timings. 
Table 2 The influence of broadcast timing on acceleration

\begin{tabular}{|c|c|c|}
\hline acceleration & $\begin{array}{c}\text { Mauchly's test } \\
\text { of sphericity }\end{array}$ & $\begin{array}{c}\text { Effect test } \\
\text { (Navigation } \\
\text { broadcast timing) }\end{array}$ \\
\hline \multirow{2}{*}{ 2-exit } & 0.282 & 0.000 \\
\cline { 2 - 3 } & $\checkmark$ & $\begin{array}{c}\text { Significant } \\
\text { difference }\end{array}$ \\
\hline \multirow{2}{*}{ 3-exit } & 0.186 & 0.000 \\
\cline { 2 - 3 } & $\checkmark$ & $\begin{array}{c}\text { Significant } \\
\text { difference }\end{array}$ \\
\hline \multirow{2}{*}{ 4-exit } & 0.487 & 0.000 \\
\cline { 2 - 3 } & $\checkmark$ & $\begin{array}{c}\text { Significant } \\
\text { difference }\end{array}$ \\
\hline
\end{tabular}

On the 2-exit, 3-exit, 4-exit expressway, the Probability distributions of LP value at the three navigation broadcast timings is shown in Figure 2

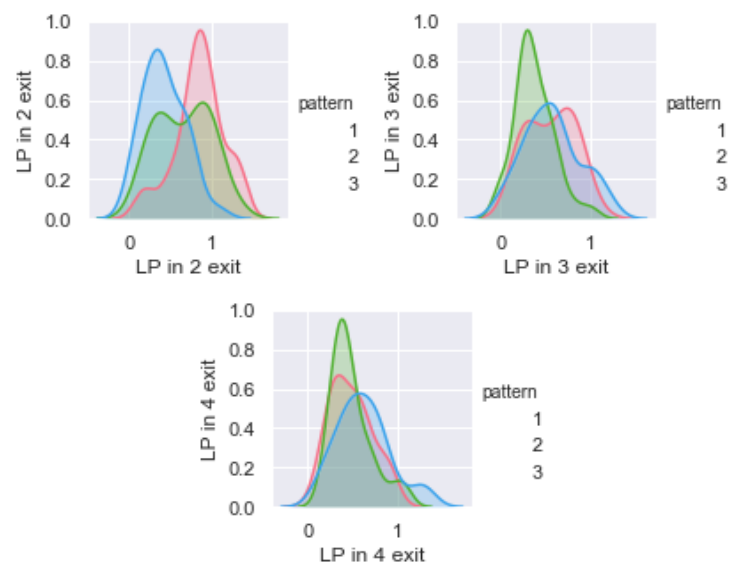

Fig 3 Probability distributions of LP

It can be seen from Table3 that the navigation broadcast timing has a significant impact on LP, that is, LP has a significant difference under different navigation broadcast timings.

Table 3 The influence of broadcast timing on LP

\begin{tabular}{|c|l|l|}
\hline \multirow{2}{*}{$\mathbf{L P}$} & $\begin{array}{l}\text { Mauchly's test of } \\
\text { sphericity }\end{array}$ & $\begin{array}{l}\text { Effect test (Navigation } \\
\text { broadcast timing) }\end{array}$ \\
\hline \multirow{2}{*}{ 2-exit } & 0.313 & 0.000 \\
\cline { 2 - 3 } & $\checkmark$ & Significant difference \\
\hline \multirow{2}{*}{ 3-exit } & 0.146 & 0.004 \\
\cline { 2 - 3 } & $\checkmark$ & Significant difference \\
\hline \multirow{3}{*}{ 4-exit } & 0.424 & 0.002 \\
\cline { 2 - 3 } & $\checkmark$ & Significant difference \\
\cline { 2 - 3 } & $\checkmark$ & Significant difference \\
\hline
\end{tabular}

The analysis shows that in all types of expressways, the timing of navigation broadcast has a significant impact on speed, acceleration, and LP. After analysis, it is found that on multi-exit urban expressway, the earlier navigation broadcast timing, the higher the average speed, and there is obvious braking and deceleration near the exit, and the absolute value of acceleration is larger. The later the navigation broadcast timing, the smaller the average speed, the less obvious braking deceleration near the exit, and the smaller the average acceleration. When the navigation broadcast timing is early, the driver thinks there is sufficient time to prepare for the lane change, so the vehicle will speed up in the early driving process. Due to the fast speed, obvious deceleration and braking behaviors are produced near the exit to facilitate lane change. At the same time, the driver will drive to the exit lane required by the navigation in advance. However, due to the complex road conditions, so the lane change frequency and the LP average value are increased. If the timing of the first announcement is late, the driver will not speed up rashly, so the average speed is low. Due to the late broadcast timing, the driver will change lanes closer to the exit, and the LP average is low.

It is concluded that the earlier the navigation broadcast timing, the higher the efficiency of the vehicle, the worse the smoothness of the vehicle, and the worse the lateral safety. The later the navigation broadcast timing, the lower the vehicle driving efficiency, the moderate driving smoothness of the vehicle, and the better lateral safety.

\subsection{Evaluation of navigation broadcast timing based on principal component analysis}

It can be seen from the above that the timing of navigation broadcast has a significant impact on driving behavior indicators, so this chapter analyzes the optimality of the navigation mode on principal component analysis. Principal component analysis is a multivariate statistical method based on dimensionality reduction. Using this method for multi-index evaluation can simplify data and reveal the relationship between variables [7].

This article includes 9 evaluation indexes about 3 aspects, and the 9 indexes are transformed into several principal components. Thus, based on the principal component, the relative score of each navigation broadcast timing is calculated. In all kinds of expressways, starting from 1900 meters upstream of the exit, with $200 \mathrm{~m}$ intervals, each index data at 10 coordinate points is used in turn to calculate the scores of different navigation broadcast timings at each coordinate point. Comprehensively consider the scoring curve of each navigation mode, so as to find the basically optimal navigation mode for each exit type expressway.

The following briefly describes the steps of the principal component analysis method to calculate the score of each navigation mode:

Step 1. Clarify evaluation indicators and evaluation objectives:

This article has three evaluation aspects: safety indicators, ride comfort indicators, and efficiency indicators. The evaluation goal is that the navigation mode with high safety, good smoothness and high efficiency is the optimal mode. The smaller the number of lane changes and the LP value, the better the lateral safety of the vehicle; the less the number of braking and the smaller the acceleration value, the better the driving smoothness; the greater the speed value, the higher the efficiency of the vehicle. 


\section{Step 2: Data standardization processing:}

First, take the reciprocal of the data negatively related to optimality, including LP mean value, steering wheel angle, braking times, lane changing times, braking times, and driving time. Second, standardize the data according to formula 1 [28].

$$
x_{i j}^{*}=\frac{x_{i j}-\overline{x_{j}}}{\sqrt{\operatorname{var}\left(x_{j}\right)}}
$$

$x_{i j}^{*}$ : The value of the $\mathrm{j}$-th indicator in the $\mathrm{i}$-th navigation mode after standardization

$x_{i j}$ : The value of the $\mathrm{j}$-th indicator in the $\mathrm{i}$-th navigation mode before standardization

$\bar{x}_{j}$ : The average value of the $\mathrm{j}$-th index before standardization

$\sqrt{\operatorname{var}\left(x_{j}\right)}$ : The standard deviation of the $\mathrm{j}$-th index before standardization

Step 3: Calculate the correlation matrix:

Based on the standardized data matrix, calculate the correlation matrix $\mathrm{R}$ according to formula 2

$$
r_{i j}=\frac{1}{n-1} \sum_{t=1}^{n} x_{t i}^{*} x_{t j}^{*}
$$

Step 4: Calculate the eigenvalues and eigenvectors of the correlation matrix:

Calculate the eigenvalue $\lambda_{c}$ of the correlation matrix $\mathrm{R}$, and the contribution rate $\alpha_{c}$ and cumulative contribution rate $\beta_{c}$ of each eigenvalue, as shown in formulas 3 and 4 .

$$
\begin{gathered}
\alpha_{c}=\left|\lambda_{c}\right| / \sum_{c=1}^{q}\left|\lambda_{c}\right| \\
\beta_{c}=\sum_{n=1}^{c} \alpha_{c}
\end{gathered}
$$

Step 5. Determine the principal components:

select $\mathrm{m}$ principal components, the cumulative contribution rate of the eigenvalues of the $\mathrm{m}$ principal components $\beta \geq 85 \%$, and the $m$ principal components $y_{1} \ldots y_{m}$ are shown in formula 5

$$
\left\{\begin{array}{c}
y_{1=}=u_{11} x_{1}^{*}+u_{12} x_{2}^{*}+\cdots+u_{1 p} x_{p}^{*} \\
y_{2=}=u_{21} x_{1}^{*}+u_{22} x_{2}^{*}+\cdots+u_{2 p} x_{p}^{*} \\
\cdots \cdots \\
y_{m=}=u_{m 1} x_{1}^{*}+u_{m 2} x_{2}^{*}+\cdots+u_{m p} x_{p}^{*}
\end{array}\right.
$$

$\left(u_{k 1} \ldots u_{k p}\right)$ Orthogonal vector of the eigenvalue $\lambda_{k}$ corresponding to the main componenty $y_{k}$

\section{Step 6. Evaluation score and ranking:}

Determine the $\mathrm{m}$ principal components $y_{1} \ldots y_{m}$ and their evaluation functions as shown in formula 6 . Each navigation mode has an evaluation score $\mathrm{F}$.

$$
F=\alpha_{1} y_{1}^{*}+\alpha_{2} y_{2}^{*}+\cdots+\alpha_{m} y_{m}^{*}
$$

$\alpha_{m}$ : Variance contribution rate of each principal component

$y_{m}^{*}:$ Score for each principal component

\section{Result}

The scores of navigation broadcast timing of the 2-exit, 3-exit, 4-exit expressway are shown in Table 4, and the trend of the three modes of navigation broadcast timing scores along the coordinate axis is shown in Figure 4 Both the 2-exit urban expressway and3-exit urban expressway, Mode 2 has the highest average score, Mode 1 is the second, and Mode 3 is the lowest. In the 3exit urban expressway, Mode 2 has the highest average score, but Mode 3 is the second, and Mode 2 is the lowest.

In all types of urban expressway, observe the scores of the three navigation modes at each coordinate point, obtaining a probability distribution figure. As shown in Figure 5, the high segment density of Mode 2 is higher, so the overall score of Mode 2 is higher.

The previous chapter has analyzed the influence of different navigation broadcast timing on the driver's driving behavior based on the speed, acceleration road, LP value and other indicators. It can be seen from the previous conclusion that the earlier the navigation broadcast timing, the higher the driving efficiency, the poorer driving smoothness and lateral safety, the later the navigation broadcast timing, the lower the driving efficiency, the moderate driving comfort, and the better lateral safety. Therefore, when the navigation broadcast timing is Mode 2, the first navigation broadcast timing is at a moderate distance from the exit, which ensures the safety and efficiency of driving. 2-exit Broadcast timing score

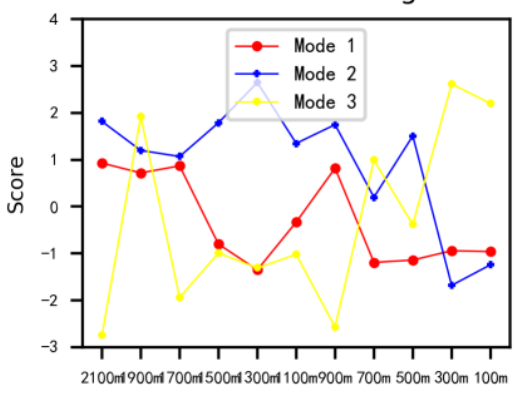

Coordinate $(m)$

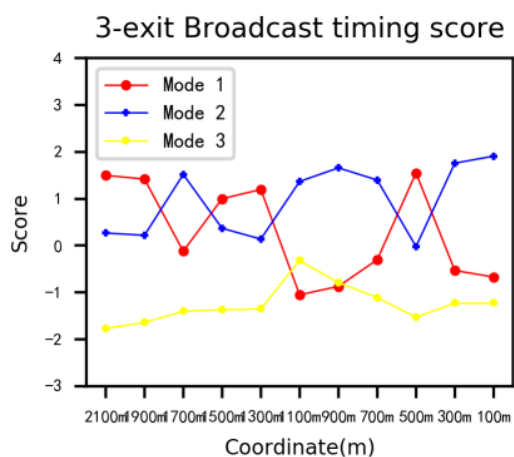

Coordinate $(\mathrm{m})$

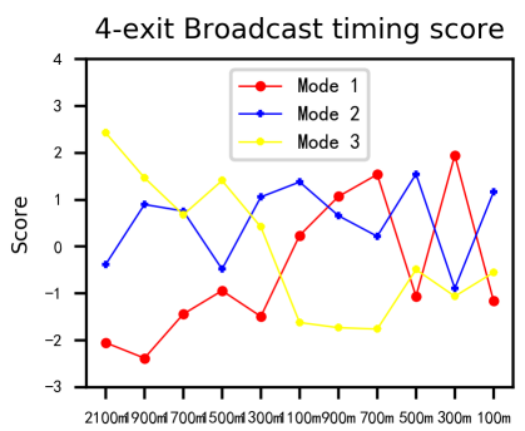

Coordinate $(\mathrm{m})$

Fig 4 Navigation mode's score trend 

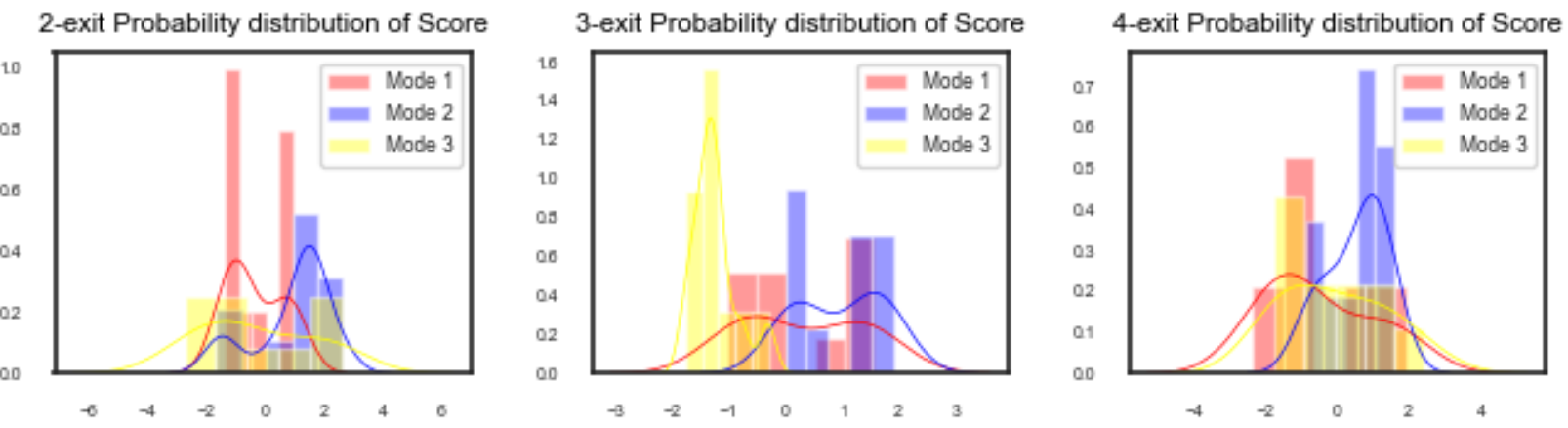

Fig 5 Probability distributions of socre

Table 4 Score of each navigation broadcast timing mode

\begin{tabular}{|c|c|c|c|c|c|c|c|c|c|c|c|c|}
\hline 2-exit & $2100 \mathrm{~m}$ & $1900 \mathrm{~m}$ & $1700 \mathrm{~m}$ & $1500 \mathrm{~m}$ & $1300 \mathrm{~m}$ & $1100 \mathrm{~m}$ & $900 \mathrm{~m}$ & $700 \mathrm{~m}$ & $500 \mathrm{~m}$ & $300 \mathrm{~m}$ & $100 \mathrm{~m}$ & $\begin{array}{c}\text { average } \\
\text { score }\end{array}$ \\
\hline Mode 1 & 0.93 & 0.72 & 0.87 & -0.8 & -1.35 & -0.33 & 0.82 & -1.19 & -1.14 & -0.94 & -0.96 & -0.306 \\
\hline Mode 2 & 1.82 & 1.2 & 1.07 & 1.79 & 2.65 & 1.35 & 1.75 & 0.2 & 1.51 & -1.68 & -1.24 & 0.9472 \\
\hline Mode 3 & -2.75 & -1.92 & -1.94 & -0.99 & -1.31 & -1.02 & -2.57 & 0.99 & -0.38 & 2.62 & 2.2 & -0.642 \\
\hline 3-exit & $2100 \mathrm{~m}$ & $1900 \mathrm{~m}$ & $1700 \mathrm{~m}$ & $1500 \mathrm{~m}$ & $1300 \mathrm{~m}$ & $1100 \mathrm{~m}$ & $900 \mathrm{~m}$ & $700 \mathrm{~m}$ & $500 \mathrm{~m}$ & $300 \mathrm{~m}$ & $100 \mathrm{~m}$ & $\begin{array}{c}\text { average } \\
\text { score }\end{array}$ \\
\hline Mode 1 & 1.5 & 1.42 & -0.12 & 1 & 1.2 & -1.05 & -0.87 & -0.3 & 1.55 & -0.53 & -0.67 & 0.284 \\
\hline Mode 2 & 0.27 & 0.22 & 1.52 & 0.37 & 0.14 & 1.37 & 1.66 & 1.4 & -0.02 & 1.76 & 1.91 & 0.963 \\
\hline Mode 3 & -1.77 & -1.64 & -1.4 & -1.37 & -1.35 & -0.32 & -0.79 & -1.11 & -1.53 & -1.23 & -1.23 & -1.249 \\
\hline 4-exit & $2100 \mathrm{~m}$ & $1900 \mathrm{~m}$ & $1700 \mathrm{~m}$ & $1500 \mathrm{~m}$ & $1300 \mathrm{~m}$ & $1100 \mathrm{~m}$ & $900 \mathrm{~m}$ & $700 \mathrm{~m}$ & $500 \mathrm{~m}$ & $300 \mathrm{~m}$ & $100 \mathrm{~m}$ & $\begin{array}{c}\text { average } \\
\text { score }\end{array}$ \\
\hline Mode 1 & -0.25 & -2.38 & -1.44 & -0.94 & -1.49 & 0.24 & 1.07 & 1.54 & -1.06 & 1.95 & -1.16 & -0.52 \\
\hline Mode 2 & -0.38 & 0.9 & 0.76 & -0.48 & 1.06 & 1.38 & 0.66 & 0.22 & 1.55 & -0.89 & 1.17 & 0.541 \\
\hline Mode 3 & 2.43 & 1.47 & 0.68 & 1.41 & 0.42 & -1.62 & -1.73 & -1.76 & -0.49 & -1.06 & -0.55 & 0.072 \\
\hline
\end{tabular}

\section{Conclusion}

This paper studies the influence of different navigation broadcast timings on driver's behavior based on the experimental data of simulated driving.

The research results prove that the navigation broadcast timing has a significant impact on each data index. Under different navigation broadcast timings, each data index has significant differences. The earlier the navigation broadcast timing, the higher the driving efficiency, the poorer driving smoothness and lateral safety, the later the navigation broadcast timing, the lower the driving efficiency, the moderate driving comfort, and the better lateral safety.

This paper also uses principal component analysis to evaluate the different modes of navigation broadcast timing. On expressways with 2-exit, 3-exit, and 4-exit, the optimal navigation broadcast timing is Mode 2, that is, the first navigation broadcast timing is $1500 \mathrm{~m}$ from the exit. Therefore, when the navigation broadcast timing is Mode 2, the first navigation broadcast timing is at a moderate distance from the exit, and the three levels of driving efficiency, driving smoothness, and lateral safety are guaranteed.

The traffic situation on urban expressways is complicated, and various voice navigation modes are likely to cause confusion to drivers. This article determines the optimal navigation broadcast timing on expressways of various types of exits, which is of great significance for reducing driver confusion and improving driving efficiency. However, there are still some imperfections in this article. It only studies the impact of broadcast timing on driving behavior, and fails to study the comprehensive evaluation model of the impact of broadcast wording on driving behavior. In the future, we will continue to study the in-depth law of the impact of navigation broadcast timing and broadcast wording on driving behavior.

\section{References}

1. Lu Min. Research on software interface design based on ergonomics[D]. Nanjing University of Aeronautics and Astronautics, 2008.

2. Zhu Wei. Human factors engineering design and evaluation of the software interface of car navigation system[D]. Harbin Engineering University, 2012.

3. Wang Ying, Zhang Wei, Wu Su. Overview of Research on Vehicle Information System and Driving Safety[J]. Science and Technology Review, 2009, 27(13).

4. Zhu Tong, Wu Ling, Lu Qiaozhen. The influence of vehicle information on driver's blink characteristics 
and load[J]. Journal of Southwest Jiaotong University, 2015, 50(3): 504-510.

5. Yun M, Zhao J, Zhao J, et al. Impact of in-vehicle navigation information on lane-change behavior in urban expressway diverge segments[J]. Accident Analysis \& Prevention, 2017, 106:53-66.

6. Qiu Hong, Jin Rufeng, Zhao Ling, et al. Using SPSS11.0 to realize variance analysis of repeated measurement data[J]. Journal of Mathematical Medicine, 2006, 19(2): 162-165.

7. $\mathrm{Gu}$ Zhenghua, Li Xuhong. Evaluation of Urban Traffic Management Planning Scheme Based on Improved Principal Component Analysis [J]. Transportation System Engineering and Information, 2004, 4(2): 51-55. 